\title{
28 Research Square \\ PAM-DOSE: A method of identifying PAMs for different CRISPR-Cas systems
}

Lianchao Tang, Fayu Yang, Xiaoxue He, Haihua Xie, Xiaoyu Liu, Junhao Fu, Haitao Xi, Xiaosheng Lu, Changbao Liu, Zongming Song, Jia Qu, Junzhao Zhao, Feng Gu

\section{Video Byte}

Keywords: PAM-DOSE, CRISPR, Cas, CRISPR/Cas, gene editing, genetics, base pair, sequence, library, signal, therapeutics

Posted Date: December 23rd, 2019

DOI: https://doi.org/10.21203/rs.2.19670/v1

License: (c) (i) This work is licensed under a Creative Commons Attribution 4.0 International License. Read Full License 


\section{Abstract}

CRSPR-Cas is a powerful gene editing tool for the therapy of human diseases But its potential for fighting human disease remains untapped Part of the problem is that different CRISPR-Cas systems use different PAM sequences to activate their editing functions and how to directly identify functional PAM sequences in human cells is still an open question Now, researchers have developed PAM Definition by Observable Sequence Excision (PAM-DOSE), a technique for identifying these secret genetic phrases It starts with using bacteria to generate a large library of random sequences on circular strands of DNA Each random sequence is flanked by tdTomato and EGFP to express red and green fluorescent proteins when implanted in a cell Only the correct sequences activate the corresponding CRISPR-Cas pair, whose function is to remove the red-protein portion So a green-only signal indicates a right answer Nextgeneration sequencing then reveals the details of the correct PAM sequence In a proof-of-principle experiment, the sequence known to activate the popular CRISPR-SpCas9 system - "NGG" - generated the strongest green signal, as expected This technique could help researchers find the right PAMs for human genome editing applications and enhance our ability to understand and fight human diseases 